Hors-série | 2015

Les élites grecques modernes, $X V I I I^{e}-X X^{e}$ siècles :

identités, modes d'action, représentations

\title{
Sappho Léondias (1830-1900)
}

« figure citoyenne majeure de la Nation »

Sappho Leondias (1830-1900), "The Great Citizen of the Nation"

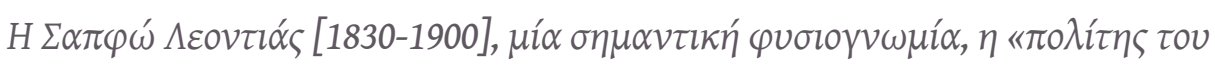

'EOvous"

\section{Louisa Christodoulidou}

\section{CpenEdition}

\section{Journals}

Édition électronique

URL : https://journals.openedition.org/ceb/5902

DOI : 10.4000/ceb.5902

ISSN : 2261-4184

Éditeur

INALCO

Édition imprimée

Date de publication : 1 mars 2015

ISBN : 978-2-85831-224-5

ISSN : 0290-7402

\section{Référence électronique}

Louisa Christodoulidou, «Sappho Léondias (1830-1900) », Cahiers balkaniques [En ligne], Hors-série I

2015, mis en ligne le 11 décembre 2015, consulté le 06 juillet 2021. URL : http://

journals.openedition.org/ceb/5902; DOI : https://doi.org/10.4000/ceb.5902

Ce document a été généré automatiquement le 6 juillet 2021.

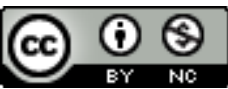

Cahiers balkaniques est mis à disposition selon les termes de la Licence Creative Commons Attribution - Pas d'Utilisation Commerciale 4.0 International. 


\title{
Sappho Léondias (1830-1900)
}

\author{
« figure citoyenne majeure de la Nation » \\ Sappho Leondias (1830-1900), "The Great Citizen of the Nation"

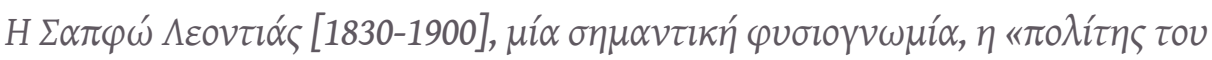 \\ 'EOvous"
}

Louisa Christodoulidou

1 La Constantinopolitaine, d'origine chypriote, Sappho Léondias ${ }^{1}$, figure majeure des lettres féminines de son époque, qui compte au nombre "des femmes qui écrivent ${ }^{2}$, a connu sa période de gloire entre le milieu et la fin du XIX ${ }^{\mathrm{e}}$ siècle, déployant une activité intense dans divers domaines: presse, enseignement, lettres. Elle est demeurée quasiment inconnue jusqu'à ce que, à une date récente, la critique se penche sur elle avec intérêt, ce qui a donné lieu, autour de son nom, à maints articles dans des revues et des volumes collectifs, des communications dans des colloques, avec des références, plus ou moins longues, systématiques ou ponctuelles à son œuvre, pédagogique essentiellement, ainsi qu'à sa production poétique mais également théâtrale.

2 Ce travail fait partie d'une plus vaste étude sur Sappho Léondias qui, tout en se proposant de mettre en lumière le jugement d'autres chercheurs sur sa personnalité et son œuvre à travers les travaux déjà publiés, entend s'appuyer essentiellement sur une recherche originale, en cours depuis des années, à une vaste échelle, et qui puise dans un matériel varié : archives, journaux, revues et journaux intimes du XIX siècle, mais également dans des écrits de Léondias elle-même, parus dans des ouvrages collectifs ou des opuscules et difficiles à trouver : traités, conférences, pièces de théâtre. Le chapitre Sappho Léondias constitue un corpus étonnamment volumineux, une somme inexploitée qui requiert une classification minutieuse et une recherche assidue, avant de l'apprécier et le commenter, car il est incontestable qu'elle écrivait sans relâche.

Le rapport de Sappho Léondias à l'écriture est multiforme et se développe à différents niveaux, vu la variété de son œuvre. Spyros Deviazis note qu'elle «était constamment occupée, enseignant, dirigeant, étudiant, écrivant ». Le travail était chez elle " presque comme une obsession » (DEVIAZIS, 1912, 73), puisqu'il constitue, en fait, un mode de vie. Par conséquent, cette relation peut être également qualifiée de passionnée dans la 
mesure où elle est liée à l'aurore de l'émancipation féminine ${ }^{3}$ et à la publication d'écrits féminins. Avec sa sœur, Émilia Kténa-Léondias ${ }^{4}$, également lettrée de son époque, elle édita le magazine féminin Eurydice (1870-873) ${ }^{5}$, qui n'eut qu'une brève existence et qui visait «à accorder une destinée égale à tout progrès intellectuel des deux sexes, toujours sans distinction de nation ni de race » (KTÉNA, 1871, 1) et à faire franchir un pas de plus vers l'articulation d'un discours public des femmes. Son ambition était

de mettre tout son cœur et toute son âme à étudier les principes de la mission de la femme, à étudier la famille, à explorer son éducation morale et physique [...] à passer en revue l'instruction publique en général, à examiner l'histoire de la femme [...], à étudier la vie et l'œuvre des représentantes illustres de son sexe [...], en collectant pieusement tout ce qu'il y a de plaisant et d'utile dans les prairies fleuries des Muses (ibidem, 2), de réfuter les représentations sociales stéréotypées des sexes et de leur différence, émanant du discours masculin dominant, et de supprimer les divisions liées au genre, en défendant le sexe féminin et son identité, et en stimulant par le biais de l'émulation l'accomplissement, le rendement de la plume féminine ${ }^{6}$ en vue de la production d'œuvres utiles à la confrérie féminine.

(кTÉNA, ibid., 2)

4 Les deux sœurs Léondias, tout comme d'autres fondatrices de revues ${ }^{7}$, ont associé la question de l'enseignement à dispenser aux jeunes filles et celle de leur éducation honnête au journalisme féminin, dans une tentative audacieuse et louable d'exprimer et de rassembler la collectivité féminine (RIZAKI, 2007, 65). C'est l'époque où l'on voit paraître des revues remarquables, dirigées par des femmes de lettres ${ }^{8}$ qui, incontestablement, se sont adonnées avec enthousiasme, à la « plume journalistique ».

\section{Pédagogue renommée}

Nous allons retracer brièvement le parcours intéressant de Sappho Léondias, à la fois en sa qualité d'auteur d'une œuvre originale, de traductrice et de pédagogue ${ }^{9}$ au prestige incontesté, et en explorant les activités parallèles et variées qu'elle a menées, qu'elles aillent ou non de pair avec l'aventure de l'écriture. Sa renommée s'est répandue partout ${ }^{10}$, car elle a réussi à instaurer des méthodes novatrices dans l'enseignement et à promouvoir des idées originales, vraiment audacieuses pour son époque. Elle a joué un rôle important dans la reconnaissance du droit des femmes à l'éducation et, de l'aveu général, joué un rôle inestimable en matière d'éducation. Les titres des conférences qu'elle a données, publiées dans des revues de l'époque, sont révélateurs de son souci passionné de l'éducation des jeunes filles : «De la formation supérieure des jeunes filles » (1857); « Des institutions de jeunes filles ou De l'éducation féminine» (1861) ; «De l'éducation des jeunes filles» (1860); «De la vocation de la femme » (1870); «De la femme» (1871); «Points de vues sur l'éducation des filles » (1872) ; «Points de vues sur la femme et son éducation» (1872) ; «Quelques paroles d'encouragement d'une femme à ses congénères » (1874); « De la véritable vocation de la femme » (1876) ; «Paroles d'or. De l'éducation féminine » (1880); « De la question féminine » (1895), entre autres, et bien sûr son poème: "À une femme instruite » (1881).

6 Ses déplacements, en tant qu'enseignante et directrice, sont impressionnants et, s'ils révèlent sa passion pour l'enseignement, ils traduisent également l'estime et la reconnaissance dont elle jouissait. Gédéon nous livre l'information intéressante selon

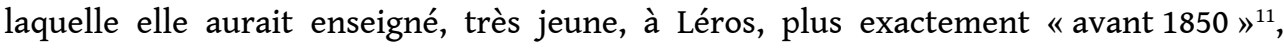


autrement dit encore adolescente. Elle s'était retrouvée dans l'île avec sa famille, lorsque son père ${ }^{12}$ fut appelé à diriger l'École Grecque d'Enseignement mutuel, tout nouvellement fondée là-bas ${ }^{13}$, de 1838 à 1845 . La famille s'installa ensuite à Chypre où, à en croire le témoignage de son élève Polyxéni Loïzias (Alitheia, 1900, 3) (Cf. LoïziAs, 1912-1920, 21 ; PYLARINOS-PARASKÉVA, 2011, 567-568), « depuis son très jeune âge, elle enseigna pendant sept ans, à titre particulier, à des jeunes filles, dans une maison de Nicosie » (LoÏZIAS, 1912-1920, 21). Loïzos Philippou laisse entendre "sur la base d'informations fournies par l'instituteur M. A. Syméonidou » $(1930,225)$ que Léondias avait enseigné dans la bourgade de Morfos. Toutefois, il ne nous a pas été possible de vérifier cette information qui semble n'avoir aucun fondement ${ }^{14}$. Ensuite, l'épouse du prince Ioannis Guikas, Alexandra, amatrice de lettres et de beaux-arts, l'invite à Samos, en 1855, pour diriger l'Institution de jeunes filles qui venait d'ouvrir ses portes (1855-1858). De 1858 à 1861, elle prend la direction de l'institution grecque de jeunes filles, fondée par Méthodios Aronis, à Smyrne. Du fait des méthodes efficaces qu'elle mettait en œuvre dans son enseignement, de sa forte personnalité, ainsi que de ses talents d'administratrice, elle fut bientôt très demandée ${ }^{15}$. Ainsi, en 1861, elle revient à Samos, répondant à l'appel du nouveau prince Aristarque, et y fonde et dirige quatre institutions de jeunes filles (1861-1863). Étape suivante de sa brillante carrière, elle devient directrice de l'Institution de Jeunes Filles d'Aghias Fotinis à Smyrne (1863-1877) et de l'Institution de Jeunes Filles Pallados (1877-1886), à Constantinople puis à nouveau de l'Institution d'Aghias Fotinis à Smyrne (1887-1891), d'où elle démissionne du fait de sa santé chancelante, après 42 ans d'une carrière d'enseignante "consacrée à l'éducation de la jeunesse féminine grecque $»^{16}$. Ainsi, en 1891, elle abandonne Smyrne pour s'installer définitivement à Constantinople (Anatolikos Astir, 1891, 247), près de sa fille, de son gendre et de ses petites-filles, Sappho et Anna ${ }^{17}$. Cependant, en dépit de sa décision de ne plus enseigner, nous lisons dans la presse (Néologos, 1891) de l'époque qu'elle reprit la direction de l'Institution de Jeunes Filles Pallas (1891-1894) et qu'en 1894 elle accepta d'être nommée comme enseignante et doyenne de l'Institution de Jeunes filles gréco-française (Néologos, 1894) à Constantinople, tout en continuant à écrire, à rédiger des articles et à prononcer des discours.

\section{« Rossignol d'Ionie au beau chant »}

7 Personnalité à multiples facettes, érudite, poétesse ${ }^{18}$ - on l'a baptisée : «le rossignol d'Ionie au beau chant »- (CHRISTOdoulidou, 2014), elle fut particulièrement prolixe puisque, outre sa production de poète, elle écrivit et traduisit une foule de manuels pédagogiques, de traités, de romans de mœurs, de nouvelles historiques ${ }^{19}$ et de pièces de théâtre ${ }^{20}$ destinées à des matinées scolaires, mais également des paraphrases, transcriptions et traductions ${ }^{21}$. Ses archives personnelles n'ont, hélas, pas été conservées et nombre de ses œuvres sont soit introuvables, soit n'ont en fin de compte pas été éditées alors que leur publication avait été annoncée. C'est le cas des Poèmes de Sappho Léondias, accompagnés d'une préface sur l'influence de la poésie sur l'esprit et les mœurs de l'homme, dont elle-même avait annoncé la parution dans la revue Néa Pandora $(1857,217)$, alors qu'elle se trouvait à Samos, dans l'intention de trouver des souscripteurs, selon la tactique habituelle de l'époque. On notera que Sappho Léondias ne recourt pas à des pseudonymes ni à l'anonymat quand elle publie ses propres textes, ainsi que le font plusieurs de ses pareilles, mais signe tantôt de ses 
initiales, rarement il est vrai et seulement à ses débuts, tantôt de son nom complet Sappho Léondias ${ }^{22}$, sous lequel elle était connue, affirmant ainsi la confiance qu'elle a en elle-même.

Elle fit deux voyages à Athènes, en 1860 et en 1883. La presse lui réserva un accueil élogieux, saluant en elle "une des Grecques les plus savantes et les plus intelligentes » (Aion, 1883, 3), la «très érudite directrice de Pallados", «célèbre dans le monde des lettres comme l'une des très rares Grecques qui se sont distinguées comme écrivains » ou encore « une Grecque distinguée, cultivée, qui, dans une Grèce asservie, honore le sexe féminin et les lettres grecques " (Néa Éphiméris, 1883, 3). Durant son séjour dans la capitale, elle eut des contacts importants avec des femmes lettrées de l'époque, dont la pédagogue Fanny Hill ${ }^{23}$. Elle fut reçue à l'université par Mistriotis qui fit son éloge aux étudiants qui se levèrent en signe de respect «envers celle qui fut l'artisan de l'éducation et de la poésie grecques " (Anatolikos Astir, 1883, 3). Elle visita l'Acropole, qu'elle salua par une ode: "À l'illustre ville d'Athènes" (Éphiméris ton Philomathon, 1860, 1492-1494), ainsi que les prisons Medressé à Plaka où elle fut surprise et chagrinée par les conditions de vie. En philanthrope qu'elle était, elle fit don d'une somme d'argent pour couvrir les besoins des détenus indigents (Néa Éphiméris, 1883, 1-2).

\section{Femme d'avant-garde}

En tant que femme d'avant-garde, elle collabora harmonieusement avec Kalliroï Parren, signant régulièrement des articles dans le Journal des Dames. Elle fonda quantité d'associations et de ligues, au sein desquelles elle mena une action soutenue. Notons son initiative de fonder l'Association "Athènes ", visant à soutenir financièrement les établissements nationaux, en l'occurrence l'Institution centrale de Jeunes Filles de Smyrne. Elle fut une helléniste distinguée, une pédagogue remarquable et une pionnière du XIX ${ }^{\mathrm{e}}$ siècle, « un Pestalozzi au féminin d'Anatolie » (LoÏZIAS, 1912-1920, 21), parlant plusieurs langues, excellente oratrice et extraordinairement cultivée. Son père Léondios Kliridis ${ }^{24}$, qui avait été l'élève de Constantinos Ikonomou, de la branche des Ikonomos de la célèbre école de Kydonia, joua un rôle déterminant à son éducation. Elle donna plusieurs conférences au Cercle littéraire de Constantinople ${ }^{25}$, privilège et honneur insigne qui lui fut presque exclusivement réservé puisqu'elle le partagea seulement avec Kalliopi Kehagia, sa redoutable rivale, la directrice de l'Institution de Jeunes Filles du Zappeion. Seules de rares femmes eurent accès à cette tribune de la forteresse dominée par les hommes.

10 Son discours, un discours de femme qui fait la part belle au genre - si peu agressif soitil - prit forme à travers ses articles, au fil de prises de parole constantes et ininterrompues. Elle fit entendre un discours public, à travers lequel elle visait non seulement à mettre en place et à soutenir l'instruction des femmes mais à forger une identité féminine. Les points de vue, très en avance sur son époque dominée par les hommes, qu'elle réussit à imposer, la conduisirent à entrer en conflit, par le biais de la presse (LÉONDIAS, 1871-1872), avec des hommes de lettres prestigieux de son temps, Ioannis Skylitsis et Andonis Isigonis, sur des questions d'égalité des sexes dans l'éducation, échanges qui mettent en lumière le caractère sexué des relations et le point de vue masculin dominant concernant la place de la femme et les restrictions auxquelles les mâles la "condamnaient». Les deux pédagogues, sous-estimant ouvertement la femme, soutenaient que, du fait de sa nature, elle devait recevoir une 
éducation en conséquence, et partant, inférieure. Selon eux, elle devait donc se contenter d'une formation encyclopédique, sa vocation n'étant de devenir ni philosophe ni politicienne mais mère, épouse et maîtresse de maison; ils alléguaient que l'entendement de la femme est inférieur à celui de l'homme. Sappho Léondias, la femme a «l'esprit masculin ${ }^{26}$, comme la définissait Parren, réagit et, à l'aide d'arguments persuasifs, réfuta les allégations des deux pédagogues dans un long traité très fouillé, qui fut publié en séries dans les journaux Amaltheia et Smyrni.

11 Elle écrit, entre autres, que dans la mesure où aucune limite n'a été fixée par les instances éducatives supérieures pour l'instruction tant des garçons que des filles ${ }^{27}$, la question d'une diversification de l'instruction selon le sexe ne se pose même pas. Skylitsis, aux points de vue duquel se range Isigonis, considère que « l'enseignement du sexe féminin doit être limité à la langue parlée ; comme la langue savante » et donc que « le grec ancien doit être banni des Institutions de Jeunes Filles, comme un épouvantail, une chose épouvantable et réprouvée " (LÉONDIAS, 1871), rétorque non sans ironie Sappho Léondias. Et elle ajoute ce commentaire sarcastique,

On n'a pas à craindre que les femmes, de par cet apprentissage, ne deviennent très savantes et très pédantes [...] au point d'inspirer dégoût et nausée à leurs affables maris.

12 Dans son chant du cygne, l'Homme et la Femme (1899), elle tente de façon très polie et fort habile, usant d'arguments théologiques (TZANAKI, ibidem, 215), d'établir l'égalité des deux sexes et de prôner la noble émulation entre ceux-ci ${ }^{28}$. Sappho Léondias juge capital que soit instaurée l'égalité dans les relations entre homme et femme, une égalité fondée sur une estime et un respect mutuels, de façon à maintenir un équilibre. L'instruction de la femme constitue à ses yeux une préoccupation prioritaire, tout comme le droit d'élire et d'être élue, en tant qu'être libre et raisonnable. Elle a la conviction que

[...] lorsqu'une société parvient à un état de civilisation plus parfait, elle reconnaît les droits des hommes et des femmes; la femme prend alors la place qui lui sied dans la cité [...] ; Elle devient citoyenne.

Et Léondias poursuit, empruntant une formule au " sage » Aimé Martin :

La place que les femmes occupent dans la société, nous donne l'histoire de la civilisation du monde.

(Amaltheia, 1877)

On ne s'étonnera pas que les interventions de Sappho Léondias, si importantes soientelles, soient restées très mesurées : cela s'explique d'abord par son tempérament ${ }^{29}$, par le milieu austère dans lequel elle évoluait et, plus généralement par le point de vue dominant et normatif sur la femme, mais cela tient également à son intime conviction que la politesse et la persuasion sont le meilleur moyen de faire aboutir les revendications. Il apparaît qu'elle n'a pas cessé un seul instant d'écrire et de lutter en faveur de l'égalité des sexes et d'une réforme de l'éducation des filles, principalement dans le monde grec d'Asie Mineure, et qu'elle a été en quelque sorte un chef de file, devenant membre de l'élite intellectuelle de son époque, où elle fut érigée en modèle et devint une référence dans maints domaines. On ne peut manquer d'être impressionnés par la place qu'elle a réussi à prendre dans le discours public, dans l'écriture et, plus généralement, par sa présence de chaque instant dans le monde des lettres. De l'aveu général, elle a laissé son empreinte de multiples manières et, à ce titre, a été qualifiée de «figure citoyenne majeure de la Nation » (O Vrettanikos Astir, 1861, 50). À en croire 
Kalliroï Parren, Sappho Léondias « fut, indubitablement, la plus sage et la plus cultivée de tous les pédagogues de ce siècle » (ibidem, 2-3).

\section{BIBLIOGRAPHIE}

\section{Bibliographie}

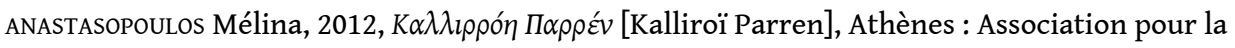
diffusion des livres utiles.

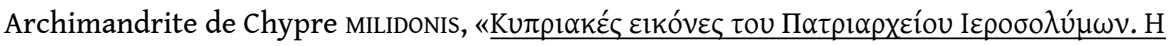

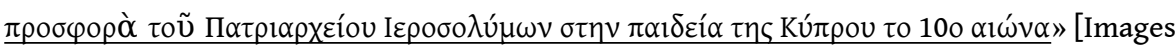
chypriotes du Patriarcat de Jérusalem ; la contribution du Patriarcat de Jérusalem à l'enseignement à Chypre, au XIX siècle], <http://www.romiosini.org.gr/bb4bf324.el.aspx>

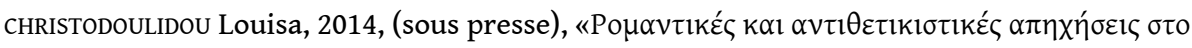

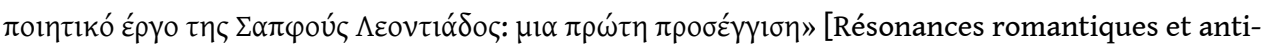
positivistes dans l'œuvre poétique de Sappho Léondias : une première approche], Estudios Neogriegos, Revista científica de la Sociedad Hispánica de Estudios Neogriegos.

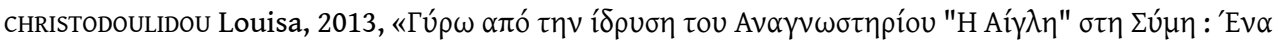

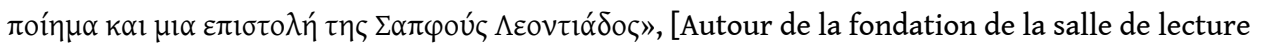
Aigli à Symi : un poème et une lettre de Sappho Léondias], Mikrophilologika, nº 33, 3-8.

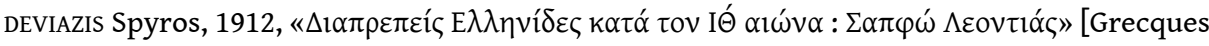
illustres au XIX ${ }^{\mathrm{e}}$ siècle : Sappho Léondias]. Elliniki Epithéorissi, $\mathrm{n}^{\circ}$ 5, 173.

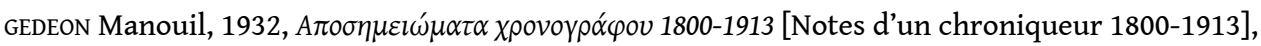
Athènes : Imprimerie Phinikos.

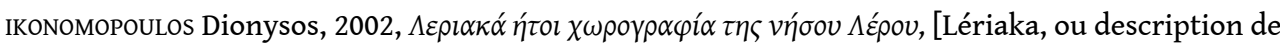
l'île de Léros] à Athènes et en Égypte 1888, Léros : Centre culturel de la municipalité de Léros.

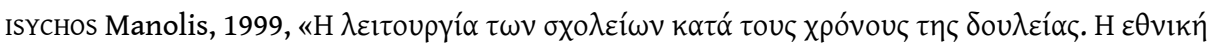

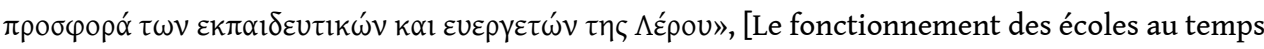
de la servitude. L'apport national des enseignants et des évergètes de Léros], Lériaka Néa, $\mathrm{n}^{\circ} 270$, 3.

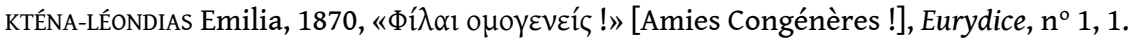

LÉONDIAS Sappho, 1899, l'Homme et la Femme; trois conférences destinées à être lues à l'Association littéraire grecque et cinq poèmes, Constantinople : Frères Yerardos.

LÉONDIAS Sappho, 1905., « Douze conseils à une fille », Constantinople : Établissements philanthropiques nationaux à Constantinople, p. 353-357.

LÉONDIAS Sappho, 1881, Chrestomathie à l'usage des Jeunes Filles, vol. B1, Smyrne : Koraïs. 
LÉONDIAS Sappho, 1877, Chrestomathie à l'usage des Jeunes Filles, vol. A', Smyrne : Phoenix. LÉONDIAS Sappho, 1876, Chrestomathie à l'usage des Jeunes Filles. vol. B2. Smyrne.

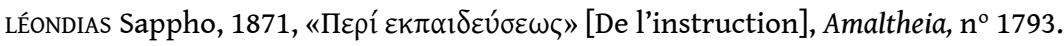

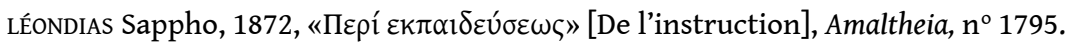

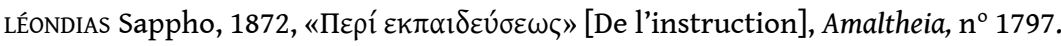

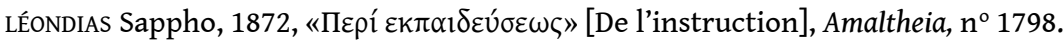

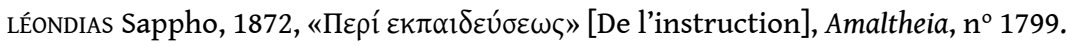

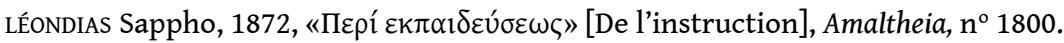

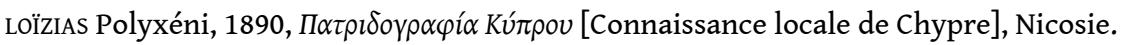

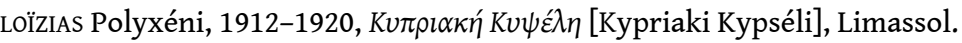
PAPADOPOUlOS Théodoros, 1991, Code des écoles de Nicosie, Nicosie : Fondation culturelle de la banque de Chypre.

PARREN Kalliroï, 1915, « Sappho Léondias », II, Journal des Dames, nº 1063, 2757-2759.

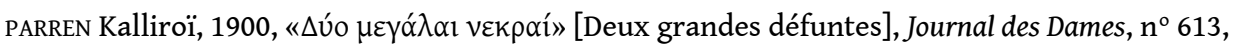
2-3.

PHILIPPOU Léon, 1930, Histoire des Lettres grecques, Nicosie : Imprimerie Thessalonique.

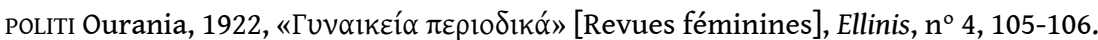

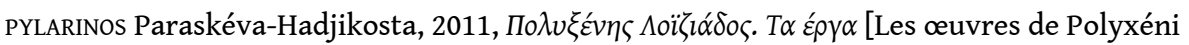
Loïzias], Nicosie : Centre de Recherches scientifiques.

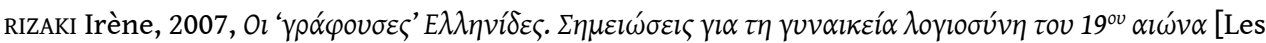
femmes qui écrivent. Notes sur les femmes lettrées du XIX siècle], Athènes : Katarti.

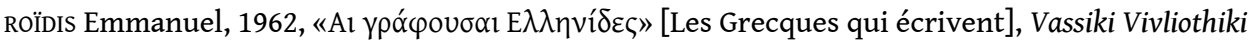
20, Athènes : éd. Aétou.

SÉRAFÉTINIDOU Alexis, 1956, «L'école de Léros en 1838 », in Archives du Dodécanèse, tome II. Athènes : Presse Sotiropoulos, p. 228-232.

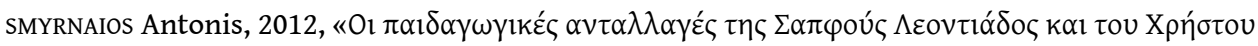

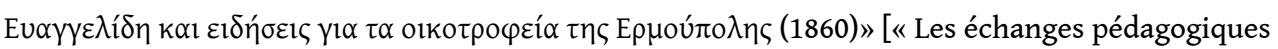
entre Sappho Léondias et Christos Evangélidis et informations sur les orphelinats d'Hermoupolis (1860) »], Microphilologika, no 31, p. 8-14.

TZANAKI Dimitra, 2007, $\Delta o v ́ \lambda \alpha \kappa \alpha \iota ~ \kappa v \rho \alpha ́$ [Esclave et maîtresse], Athènes : Savvalas.

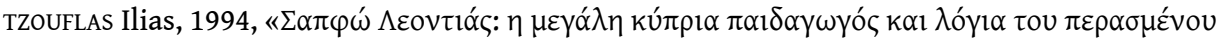

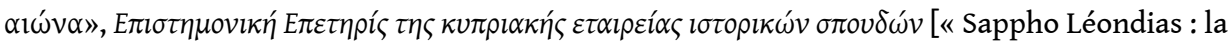
grande pédagogue et femme de lettres chypriote du siècle dernier ", in Annuaire scientifique de la société chypriote d'études historiques], tome II. Nicosie : Société chypriote d'études historiques, p. 243-274. 


\section{Journaux}

Alitheia, $\mathrm{n}^{\circ}$ 991, 10 mars 1900, Limassol : 3.

Aion, $\mathrm{n}^{\circ}$ 4297, 18 octobre 1883, Athènes : 3 .

Amaltheia, $\mathrm{n}^{\circ} 331,9$ mars 1877, Smyrne.

Anaplassis, $\mathrm{n}^{\circ}$ 149, 15 février 1894, Athènes : 2048.

Anatolikos Astir, $\mathrm{n}^{\circ}$ 31, 17 juillet 1891, Constantinople : 247 .

Néa Éphiméris, nº 288, 19 octobre 1883, Athènes : 3.

O Vrettanikos Astir, $\mathrm{n}^{\circ} 28,16$ janvier 1861.

\section{NOTES}

1. Elle est née et décédée à Constantinople. Ilias Tzouflas a mis au jour l'acte de mariage de Léondias, qu'il a publié tel quel, et qui mentionne pour elle comme lieu de naissance Méga Revma à Constantinople et non pas Moutoullas, comme l'avait prétendu son élève Polyxéni Loïzias $(1890,14)$. Il fait également mention de son âge l'année de son mariage, ce qui permet de conclure qu'elle est née en 1830 (1994, 245-246). Son père, originaire de Marathasa ou Myrianthousa, était le célèbre instituteur et helléniste, Léondios Kliridis, sa mère, Sofia Kliridis, et sa sœur, Émilia Kténa-Léondias. Elle épousa le grand négociant Konstantinos Kalvokoressis (1824-1874), un Constantinopolitain originaire de Chios. Ils eurent quatre enfants dont trois moururent bébés ou en bas âge. Corinna, sa fille unique, épousa Stéfanos Narlis, médecin, député de Gallipoli au Parlement ottoman (1908) qui fut élu sénateur avec les Libéraux, en avril 1929, lorsque Elefthérios Venizélos était Premier ministre. Corinna se suicida en 1902, deux ans après la mort de sa mère.

2. Formule employée pour la première fois par Emmanuel RoÏDIS $(1962,5)$.

3. Encore que LÉONDIAS écrive : «je n'admets pas le mot émancipation car il signifie affranchissement d'un esclave [...] ; la femme [...] est par nature égale à l'homme et d'égale valeur ; elle n'est en rien esclave ni soumise » $(1899,85-86)$.

4. Si l'on en croit Ourania POLITI, S. Aliberti soutient que S. Léondias elle-même lui avait confié qu'en 1885, elle avait été hébergée dans la maison de Zappas à Vresthéni : "Grâce à mon poste de directrice de l'Institution de jeunes filles, j'ai laissé entendre que l'éditrice de ma revue, "Eurydice”, était ma sœur. Mais la revue m'appartient » (1922, 105-106).

5. « Eurydice a été la première voix sérieuse de l'action féminine de l'Hellénisme, dans notre journalisme » (DEVIAZIS, 1912, 172-173).

6. Irini RIZAKI écrit qu'Eurydice est « un terrain privilégié d'expérimentation pour la culture et la diffusion de l'écriture féminine » $(2007,66)$.

7. C'est Eufrossini SAMARTzIDOU qui a publié la première revue, Kypséli [La Ruche] (1845).

8. Thaleia (1867) de Pénélopi LAzARIDOU.

9. «Sappho Léondias a pris une place enviée parmi les maîtres de la Race, car elle a œuvré avec sincérité pour donner aux femmes une éducation qui sied à des Grecques. 
Et elle fait œuvre d'excellente pédagogue. Elle a voué toute sa vie à l'éducation féminine, elle portait dans son cœur et avait sans cesse à l'esprit ses élèves et le progrès de son sexe. » (DEVIAZIS, 1912, 172-173).

10. À l'occasion de la fondation de la Salle de lecture "Aigli », en 1872, à Symi, les habitants progressistes de l'île lui demandèrent de leur présenter la personne adéquate pour diriger l'institution de jeunes filles de Symi nouvellement fondée. Léondias proposa Éléni Aleiféri (CHRISTODOULIDOU, 2013, 6). À noter également que, partout où elle allait, elle séduisait tout le monde par ses talents d'oratrice. Christos Evangelidis, directeur du «Lycée grec » de Syros, dans son journal intime encore inédit, qualifie Sappho Léondias, alors âgée de trente ans, en ces termes: "dotée d'une éducation satisfaisante et de convictions admirables » (SMYRNAIOS, 2012, 9).

11. «Sappho Léondias s'était taillé une réputation suite à la tâche d'enseignante qu'elle avait accomplie à Léros (avant 1850) et à Samos » (GÉDÉON, 1932, 135).

12. Cf. La réimpression du livre de IKonomopoulos, $(2002,121)$ et celui de IsYchos, $(1999$, 3).

13. On proposa à L. Kliridis les maisons de Maria Marouda et de Madame Kali Karavassili, respectivement comme école et comme logement, ainsi qu'il était prévu dans son contrat. Sur l'école fut gravée l'inscription suivante: "source de la sagesse et dispensatrice de vie. Les habitants de Léros honorent les Muses. 1838 A Léros » (sÉrAfÉtinidou, 956, 228-232). À l'endroit où se dresse aujourd'hui le Musée archéologique se trouvait la maison qui avait alors fait office d'école. L'inscription ci-dessus a été gravée sur les parois de la fontaine.

14. À en croire Loïzos Philippou, avant 1840 et avant l'ouverture d'une école véritable à Morfos, Léondios Kliridis, y avait enseigné la littérature grecque (op. cit., 230).

15. M. G. Siakallis, au nom de l'Éphorie des établissements d'enseignement de Nicosie, adressa (le 27 mai 1869) une lettre à Sappho Léondias pour la prier d'apporter sa contribution à l'enseignement à Chypre, en acceptant de diriger l'Institution de jeunes filles de Nicosie, après que Carolina Smoltze eut démissionné de son plein gré (PAPADOPOULOS, 1991, 143).

16. À l'occasion de sa mort, nous lisons dans le journal chypriote Alitheia « Un demisiècle d'un travail assidu et inlassable, une vie entière consacrée à l'éducation de la jeunesse féminine grecque !» (Alitheia, 1900, 3).

17. Dimitra TZANAKI, dans son étude par ailleurs remarquable $(2007,254)$, commentant les « Douze conseils à une fille ", mentionne par inadvertance Anna comme étant la fille de Léondias et non sa petite-fille. À noter que l'article ci-dessus fut publié pour la première fois après la mort aussi bien de Sappho que de Corinna, à l'initiative et aux bons soins de St. Narlis.

18. À ce jour, j'ai réuni cent soixante-dix de ses poèmes, soit épars dans la presse de l'époque, soit publiés dans divers volumes et je poursuis ma collecte. Je connais l'existence d'autres poèmes par des annonces ou des références dans la presse du $\mathrm{XIX}^{\mathrm{e}}$ siècle, mais il ne m'a pas encore été possible de les repérer.

19. Alors qu'elle se trouve à Syros, est annoncée dans le journal Enossis $\left(\mathrm{n}^{\circ} 336\right.$, 3 septembre 1860) d'Ermoupolis la parution des Pages choisies de Sappho Léondias, qui

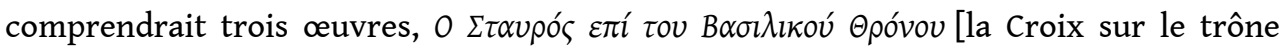

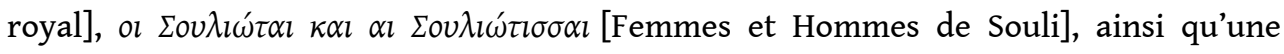


paraphrase de la tragédie d'Eschyle, les Perses. Je remercie mon collègue Andonis Smyrnaios de m'avoir communiqué cette annonce.

20. Ses pièces de théâtre connues et faciles à trouver sont les $\Pi \alpha \rho \alpha \sigma \tau \alpha ́ \alpha \sigma \varepsilon ı \zeta \delta \rho \alpha \mu \alpha \tau \imath k \alpha i$

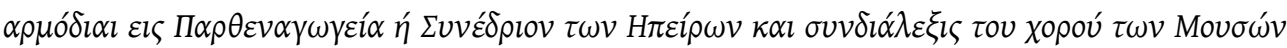

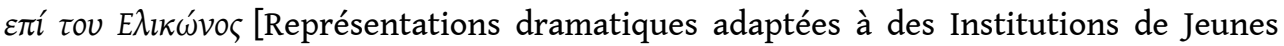
Filles, ou Congrès des Continents et assemblées du chœur des Muses sur l'Hélicon], Smyrne, Imprimerie d'Amaltheia, 1871, $\kappa \alpha \iota H \pi \alpha \tau \rho i ́ \varsigma \delta v \sigma \varphi o \rho o v ́ \sigma \alpha$ [la Patrie qui renâcle]. À noter que, par un encart non encore inexploité de l'Anaplassis, à la rubrique «Bulletin de la Bibliothèque » $(1894,2048)$ nous apprenons qu'entre autres pièces déjà connues, «Madame Sappho Léondias [a fait don] de ses écrits» Xopós $\Omega \kappa \varepsilon \alpha v i ́ \delta \omega v$ [Danse des Océanides] afin de constituer la bibliothèque de la revue, œuvre qui toutefois fait défaut.

21. Comme, entre autres, les Perses d'Eschyle et Esther de Racine. Nous lisons que «le 17 novembre 1851, le Métropolite de Pétra, Mélétios, épitrope du Patriarche de Jérusalem, informe l'instituteur Léondios à Chypre que le Patriarcat contribue à hauteur de cinq cents (500) piastres par le biais du Vicaire général du Patriarcat à Chypre, l'archimandrite Narkissos, afin d'inscrire la "Communauté du Saint Sépulcre" au nombre des souscripteurs du livre que va éditer sa fille Sappho Léondias. Il s'agit de la traduction en français de la tragédie de Racine Esther » (Archimandrite de Chypre Milidonis, <http://www.romiosini.org.gr/bb4bf324.el.aspx>.

22. Une fois seulement, elle signa Sappho Léondias Kalvokoressi quand elle publia un poème à l'occasion de la disparition de son mari.

23. Lors de sa visite, Sappho Léondias aborde le chapitre de Kehagia, probablement en la calomniant, mais Hill exprime son indignation face à la polémique lancée contre son ancienne collaboratrice. Cf. Lettre de Hill à Kehagia, 15/27 novembre 1877, Archives de l'école Hill et Efi Canner, 2012, 125.

24. Son nom de famille est dérivé du petit nom de son père. Elle a voulu ainsi l'honorer, en signe de gratitude pour l'éducation qu'il lui a dispensée.

25. Entre autres conférences, signalons «La femme dans la tragédie grecque antique », "Comment il convient d'éduquer la femme en vue de sa double vocation ", "Quel est le fondement de l'éducation morale», «De l'éducation des femmes chez nous depuis 1853 ", « Des beautés homériques », «Le nouveau programme d'éducation ».

26. «Sappho Léondias n'était pas une femme à faire du bruit ni à se mettre en avant. Mesurée, humble, mobilisant tout son esprit masculin et son fort tempérament, elle a œuvré sans ambition, avec un seul et unique objectif: se rendre utile à son sexe, transmettre ses connaissances et ses principes, qui étaient des principes de vérité et de vertu. Et elle y a réussi. » (PARREN, 1900, 2-3).

27. Dans ce traité, Sappho Léondias divulgue qu'elle a déjà commencé depuis cinq ans à écrire une encyclopédie pour les jeunes filles, dans les moments perdus que lui laissent ses obligations professionnelles. Elle fait manifestement référence à la Chrestomathie à l'usage des Jeunes Filles, dont seuls trois tomes furent finalement publiés au lieu des dix qu'elle avait initialement annoncés.

28. Parallèlement, elle s'étonne des théories stupides qui font dépendre l'intelligence de la quantité de masse cérébrale et, pour les réfuter, elle avance le point de vue du savant Waldeyer, qui donne comme exemple le cerveau de l'éléphant, plus volumineux 
que celui de l'homme alors que «celui-ci n'est pas plus intelligent que l'homme » (LÉONDIAS, ibidem, 76).

29. L'opinion initialement négative que se forma d'elle Kalliroï Parren, lors de leur première rencontre à Constantinople est révélatrice (1915, 2757-2759). Cf. ANASTASOPOULOS, 201, 972-98.

\section{RÉSUMÉS}

Dans ce travail, qui s'appuie sur une recherche originale et un matériel d'archives déjà constitué, nous nous pencherons sur le cas de la constantinopolitaine d'origine chypriote Sappho Léondias, et sur les domaines dans lesquelles elle a déployé son activité : presse, enseignement, lettres. Léondias a déployé l'essentiel de son activité à partir de la fin du XIX ${ }^{\mathrm{e}}$ siècle, "toujours enseignant, dirigeant, étudiant, écrivant ".

Pédagogue majeure et pionnière $\mathrm{du} \mathrm{XIX}^{\mathrm{e}}$ siècle, parlant plusieurs langues, extraordinairement cultivée, elle était la sœur d'Emilia Kténa-Léondias avec laquelle elle édita le magazine féminin Eurydice. Personnalité à multiples facettes, érudite, poétesse (on l'a baptisée : «le rossignol d'Ionie au beau chant »), elle fut particulièrement prolixe puisque, outre la poésie, elle a écrit et traduit une foule de manuels pédagogiques, de nouvelles et de pièces de théâtre. On lui doit notamment la traduction d'Esther de Racine.

Nous retiendrons surtout sa contribution à l'éducation des jeunes filles et le rôle qu'elle a joué pour instaurer de nouvelles idées et des méthodes d'avant-garde dans l'enseignement. Son discours, un discours de femme qui fait la part belle au genre sur des questions d'égalité des sexes dans l'éducation, la conduisirent à entrer en conflit avec des hommes de lettres prestigieux de son temps, par le biais de la presse.

Il apparaît qu'elle n'a pas cessé un seul instant d'écrire et de lutter en faveur de l'égalité des sexes et d'une réforme de l'éducation des filles, principalement dans l'Hellénisme de l'Asie Mineure et qu'elle a été en quelque sorte un chef de file, ce qui la range dans l'élite de son époque, où elle fut érigée en modèle et devint une référence dans maints domaines. De l'aveu général, elle a laissé son empreinte de multiples manières et à ce titre a été qualifiée de «citoyenne majeure de la Nation ».

This paper, based on primary research and archival material, will examine Sappho Leondias' significant contributions to three areas of achievement: Education, Press, and Greek Letters. A very influential 19th century woman of Cypriot origin, Sappho lived in Constantinople where her work flourished from the mid to the late Nineteenth century. Highly educated and multi-lingual she was "always busy teaching, directing and studying". Sappho was the sister of the well-known Emilia-Ktena Leondias with whom she published the influential women's periodical Eurydice (1870-1873). An intellectual, Sappho was also a multifaceted personality with a love for writing poetry and therefore described as "the melodious Ionian nightingale". Also a prolific writer she was dedicated to writing and translating a plethora of pedagogical manuals, short stories and theatrical performances. One of her major works was her translation of Racine's Esther.

A zealous advocate of female education Sappho Leondias played an important role in supporting the female identity as well as in establishing more progressive ideas and methods in teaching. Her active roles in promoting gender equality in education brought her into conflict, especially 
in the press, with prominent Greek scholars of her time. Fighting relentlessly for equal education at home she also supported Greeks of the "Diaspora". A great leader of her time she ranks among the elite female personages as indicated by the honour bearing title: "Great Citizen of the Nation".

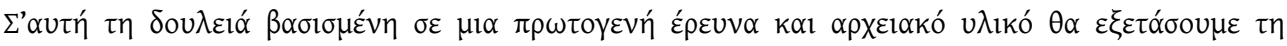

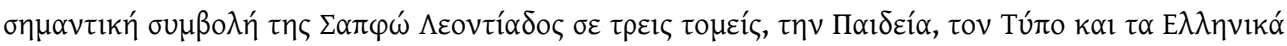

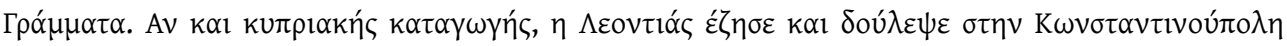

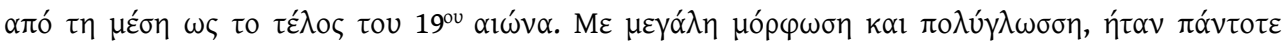

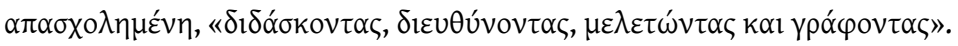

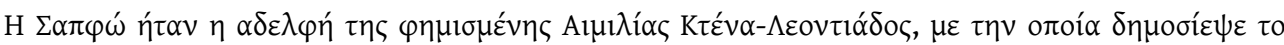

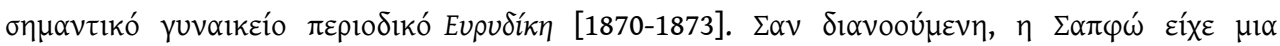

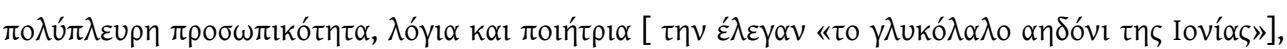

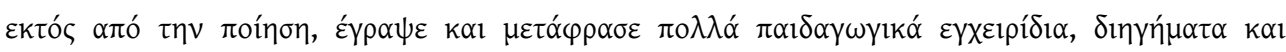

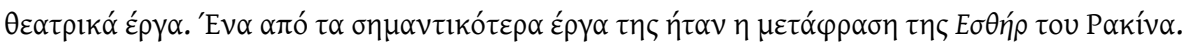

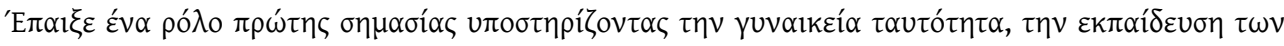

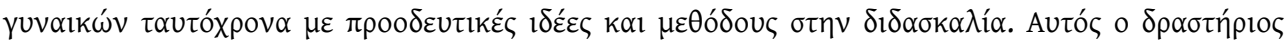

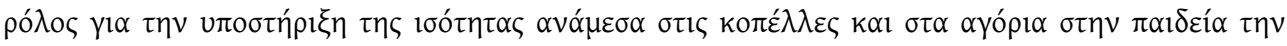

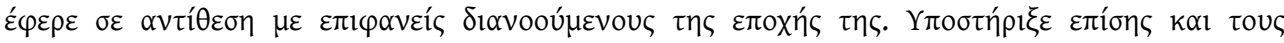

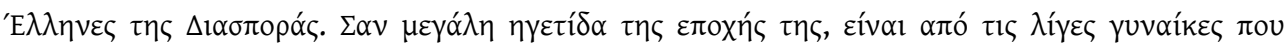

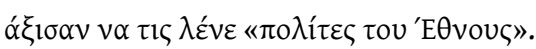

\section{INDEX}

Mots-clés : Léondias Sappho (1830-1900), Léondias Sappho (1830-1900), éducation, féminisme Thèmes : Littérature grecque

Keywords : Leondias Sappho (1830-1900), Education, Feminism, Constantinople, Greece, Nineteenth century, Greek literature

motsclesmk ЛЕОНДИАС САФО (1830-1900), ОБРАЗОВАНИЕ, ФЕМИНИЗМОТ, ЦАРИГРАД, ГРЦИЈА, ДЕВЕТНАЕСЕТТИОТ ВЕК, ГРЧКАТА ЛИТЕРАТУРА

motsclestr Leondias Sappho (1830-1900), Eğitim, Feminizm, İstanbul, Yunanistan, Ondokuzuncu yüzyıl, Yunan Edebiyatı

Index géographique : Constantinople, Grèce

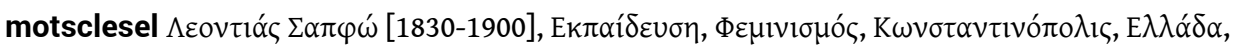

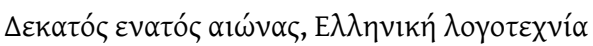

Index chronologique : dix-neuvième siècle

\section{AUTEUR}

\section{LOUISA CHRISTODOULIDOU}

Université de l'Égée 